\title{
INDICATIONS FOR GENETIC TESTING FOR DRAVET SYNDROME
}

Researchers at the Cincinnati Children's Medical Center, $\mathrm{OH}$ investigated the predictive value of features of Dravet syndrome, as defined by the International League Against Epilepsy, as criteria for a positive SCN1A gene mutation in a cohort of consecutively tested children. SCN1A mutations were present in $16(23 \%)$ of 69 children tested. More than 4 ILAE criteria demonstrated $100 \%$ sensitivity. Seven criteria (resistance to multiple antiepileptic drugs, multiple seizure types, abnormal EEG, exacerbation with hyperthermia, normal development before seizure onset, seizure onset before age 1 year, and psychomotor retardation) were present in $>85 \%$ of mutation positive patients. Three criteria best predicted a mutation in SCN1A: 1) exacerbation with hyperthermia, 2) normal development before seizure onset, and 3) appearance of ataxia, pyramidal signs, or interictal myoclonus. The first 2 criteria were most specific. Testing children who meet $>4$ criteria would constitute a high sensitivity strategy. The use of appropriate clinical criteria to define a test population for mutations of SCN1A should allow earlier recognition of the diagnosis and initiation of optimal antiepileptic therapy and family counseling. (Fountain-Capal JK, Holland KD, Gilbert DL, Hallinan BE. When should clinicians order genetic testing for Dravet syndrome? Pediatr Neurol November 2011;45:319-323). (Respond: Dr Fountain-Capal, Division of Neurology, Cincinnati Children's Medical Center, 3333 Burnet Ave, MLC 2015, Cincinnati, OH 45229. E-mail: jamiekcapal@gmail.com).

COMMENT. Recurrence of fever precipitated seizures (especially when triggered by vaccination) followed by psychomotor regression, in an infant with previously normal development should signal a diagnosis of Dravet syndrome and lead to SCN1A genetic testing and more effective therapy. The development of anticonvulsant drugs more effective in prevention of recurrent complex febrile seizures may be of potential benefit in Dravet patients. Phenytoin and carbamazepine, found to exacerbate Dravet seizures are also contraindicated in febrile seizure patients. Valproate, effective in Dravet patients, is also effective in prevention of febrile seizures. Stiripentol (Diacomit), a GABAergic agent, approved as an orphan drug in 2007 in Europe for adjunctive therapy in Dravet syndrome, and clobazam, combined with valproate, is recommended following two independent randomized placebo-controlled trials. Topiramate and ketogenic diet are alternatives in pharmaco-resistant cases. (Chiron C, Dulac O. The pharmacological treatment of Dravet syndrome. Epilepsia April 2011;52 (Suppl 2):7275)(Chiron C. Current therapeutic procedures in Dravet syndrome. Dev Med Clin Neurol April 2011;53 (Suppl 2):16-18).

Clobazam in Lennox-Gastaut syndrome is evaluated as an adjunctive therapy and found effective in reducing mean weekly drop seizure rates over 12 weeks. (Ng YT et al. Neurology October 14, 2011;77:1473-1481). Somnolence, pyrexia, upper respiratory infections, and lethargy were the most frequent adverse events reported for clobazam. 